\title{
CT appearance of disseminated peritoneal adenomucinosis
}

\author{
R. E. Bechtold, ${ }^{1}$ M. Y. M. Chen, ${ }^{1}$ B. W. Loggie, ${ }^{2}$ S. L. Jackson, ${ }^{3}$ K. Geisinger ${ }^{3}$ \\ ${ }^{1}$ Department of Radiology, Wake Forest University School of Medicine, Medical Center Boulevard, Winston-Salem, NC 27157-1088, USA \\ ${ }^{2}$ Department of Surgery, Wake Forest University School of Medicine, Medical Center Boulevard, Winston-Salem, NC 27157-1088, USA \\ ${ }^{3}$ Department of Pathology, Wake Forest University School of Medicine, Medical Center Boulevard, Winston-Salem, NC 27157-1088, USA
}

Received: 19 September 2000/Accepted: 18 October 2000

\begin{abstract}
Disseminated peritoneal adenomucinosis (DPAM) is a relatively rare cause of pseudomyxoma peritonei, marked by peritoneal lesions with abundant extracellular mucin and little cytologic atypia among exfoliated tumor cells, and usually associated with appendiceal adenomas. Peritoneal mucinous carcinomatosis (PMCA) also causes diffuse peritoneal tumor but is marked by neoplastic mucinous epithelium with cytologic features of carcinoma and associated with appendiceal, colon, gastric, or small bowel carcinoma. Compared with PMCA, DPAM has a different distribution of disease and a significantly better prognosis. DPAM is characterized by the lack of lymph node involvement, with primarily superficial peritoneal involvement, and a relatively benign, relapsing course over many years. Dominant primary masses may not be evident in DPAM. Despite considerable overlap in the two main causes of pseudomyxoma peritonei, there are features of the two that may help differentiate between them. We present the computed tomographic appearance of DPAM in a series of seven cases and emphasize characteristics of the disease.
\end{abstract}

Key words: Pseudomyxoma peritonei-Peritoneum, neoplasm-Peritoneum, CT.

Pseudomyxoma peritonei is a clinical condition in which gelatinous ascites and peritoneal implants proliferate in the peritoneal cavity. Pseudomyxoma peritonei has been described in association with benign and malignant tumors arising from many different organs. To clarify the definition of pseudomyxoma peritonei, multifocal peritoneal mucinous processes can be divided into three categories [1-3]. One is

Correspondence to: R. E. Bechtold benign disseminated peritoneal adenomucinosis (DPAM). Another is malignant peritoneal mucinous carcinomatosis (PMCA). A third, small category is intermediate between the other two. These three diseases have different distributions and prognoses. We contrast DPAM and PMCA as depicted on computed tomography (CT).

\section{Materials and methods}

We reviewed medical records of consecutive patients with mucinous peritoneal neoplasms treated by the Surgical Oncology section at our institution over 22 months, from April 1997 to February 1999. From this group, seven patients with a diagnosis of DPAM and a CT study before the treatment were identified. A randomly acquired but otherwise similar cohort of seven patients who had a CT study before treatment and PMCA was defined from this same overall patient population. The diagnoses of all patients were made from specimens obtained at laparotomy and consisted of DPAM or PMCA. No intermediate form was encountered.

The seven patients with DPAM were 39 to 64 years old (mean $=49$ years). Three were male, and four were female. Four patients with DPAM had a history of appendiceal adenoma. The remaining three patients with DPAM had no history of appendiceal adenoma in their records. The seven patients with PMCA were 36 to 55 years old (mean $=47$ years). Four were male, and three were female. Primary tumors in patients with PMCA included appendiceal mucinous adenocarcinoma in five patients, small bowel adenocarcinoma in one patient, and mucinous adenocarcinoma from an unknown site in one patient.

Preoperative CT scans in all patients were obtained using General Electric HiSpeed Advantage or CT/i CT scanners. Oral contrast material, $700 \mathrm{~mL}$ of diatrizoate meglumine, (Gastroview, Mallinckrodt, St. Louis, MO, USA) was administered. Power injection (Medrad, Pittsburgh, PA, 
Table 1. Radiographic features of DPAM versus PMCA

\begin{tabular}{|c|c|c|}
\hline & DPAM & PMCA \\
\hline Pleural effusion, pleural rind & None & $14 \%(1 / 7)$ \\
\hline \multicolumn{3}{|l|}{ Ascites } \\
\hline Large amount ${ }^{a}$ & $71 \%(5 / 7)$ & $43 \%(3 / 7)$ \\
\hline Small amount ${ }^{a}$ & $28 \%(2 / 7)$ & $43 \%(3 / 7)$ \\
\hline Loculated & & $14 \%(1 / 7)$ \\
\hline Omental cake & None & $57 \%(4 / 7)$ \\
\hline \multicolumn{3}{|l|}{ Hepatic scalloping } \\
\hline Typical & $43 \%(3 / 7)$ & None \\
\hline Minimal & None & $43 \%(3 / 7)$ \\
\hline Parenchymal invasion (liver, spleen) & $57 \%(4 / 7)$ & $57 \%(4 / 7)$ \\
\hline Reticular infiltration of the mesentery & $14 \%(1 / 7)$ & $14 \%(1 / 7)$ \\
\hline Peritoneal mass (implants) & $71 \%(5 / 7)$ & $57 \%(4 / 7)$ \\
\hline Lymph node & None & $14 \%(1 / 7)^{b}$ \\
\hline Mass calcification & $29 \%(2 / 7)$ & None \\
\hline Primary lesion present & None & $14 \%(1 / 7)$ \\
\hline
\end{tabular}

${ }^{a}$ Large amount, abdomen full of fluid; small amount, abdomen partly filled with fluid (subjective categorization)

${ }^{b}$ Periaortic lymph node

USA) of a monophasic bolus of $125 \mathrm{~mL}$ of ioversol (Optiray 350, Mallinckrodt Medical) at a rate of $2 \mathrm{cc} / \mathrm{s}$ was performed, and scanning was begun 55-70 s after injection to coincide with the portal venous phase. Images were obtained using a spiral technique, including 1-s scans, 7-mm-thick sections, and a 2:1 pitch. CT studies were obtained of the lung bases, abdomen, and pelvis in all patients. CT studies were obtained of the entire chest in 11 patients. Interpretation was performed from hardcopy film.

CT findings were procured from the preoperative CT report to avoid bias based on knowledge of the pathologic results. Two of us reviewed original reports and actual films to confirm the accuracy of the radiologic findings. No additional findings were obtained from this retrospective review. CT findings such as ascites, omental cake, hepatic scalloping, parenchymal invasion, peritoneal mass, infiltration of the mesentery, calcification of the mass lesion, enlarged lymph nodes, presence of pleural effusion, and presence of primary lesion were recorded and correlated between the two groups.

The quantity of ascites was subjectively determined to be a large amount if the abdomen was full of fluid and a small amount if the abdomen was partly filled with fluid. Hepatic scalloping was determined to be minimal if the depth of scalloping wave was less than $5 \mathrm{~mm}$ and typical if the depth of the scalloping wave was greater than $5 \mathrm{~mm}$.

\section{Results}

The relative frequency of each of the major CT findings from patients with both DPAM and PCMA is recorded in Table 1. There is considerable overlap in some of the features of these two diseases, but certain findings bear consideration. Coexistent disease in the chest was seen only in cases of PMCA (Fig. 1). The most common manifestations in the chest included pleural effusion and pleural rind.

Abdominal manifestations overlapped more, but certain differences still existed. CT features that did not appreciably differ from one another include hepatic scalloping (Fig. 2), parenchymal organ invasion (Figs. 1, 3), infiltrative changes in the mesentery (Figs. 4, 5), and peritoneal nodules. Features in our series that may differ include the presence of large volumes of mucinous ascites and calcification in the masses as they were more often present in DPAM (Figs. 2, 6). The presence of omental cake (Fig. 3), lymphadenopathy (Fig. 3), and visualization of a primary mass or peritoneal implant were common in the PMCA. DPAM occurred without the findings of an omental cake and more often with typical hepatic scalloping.

\section{Discussion}

Multifocal peritoneal mucinous tumors can arise from several different entities, and the classification of the origins of peritoneal tumor and pseudomyxoma peritonei in particular has evolved in time. Secondary peritoneal malignancy arises from many intraabdominal and extraabdominal sources, of which appendiceal conditions, both benign and malignant, are one important category. Appendiceal processes include mucocele, mucinous cystadenoma, and mucinous cystadenocarcinoma. Some investigators have classified mucoceles as arising from hyperplasia, mucinous cystadenoma as arising from hyperplasia with epithelial atypia, and mucinous cystadenocarcinoma as associated with stromal invasion [4]. Extraappendiceal spread of peritoneal disease and pseudomyxoma peritonei suggest malignancy [5]. However, the pathogenesis of pseudomyxoma peritonei is controversial [5], and its origin has been variably attributed to spillage of malignant mucin [6] and to spread of a malignant mucocele [7]. These explanations have in common that pseudomyxoma peritonei is a histologically malignant condition. However, pseudomyxoma peritonei does not necessarily arise in the setting of malignancy. According to Ronnett et al. [1, 2], pseudomyxoma peritonei only occurs in DPAM, not in PMCA; therefore, the features of pseudomyxoma peritonei and DPAM overlap because pseudomyxoma peritonei is part of DPAM.

Ronnett et al. [1, 2] first attempted to clarify the confusing etiologies of diffuse peritoneal tumors by categorizing the disease process, which produced multifocal peritoneal mucinous tumors. These processes included DPAM and PMCA as well as PMCA with discordant or intermediate features. They concentrated on the pathologic features of these diseases.

Histologic criteria of DPAM include lakes of mucin with scant strips of abnormal glandular epithelium. The epithelial cells are relatively bland. In many instances, the pools of 

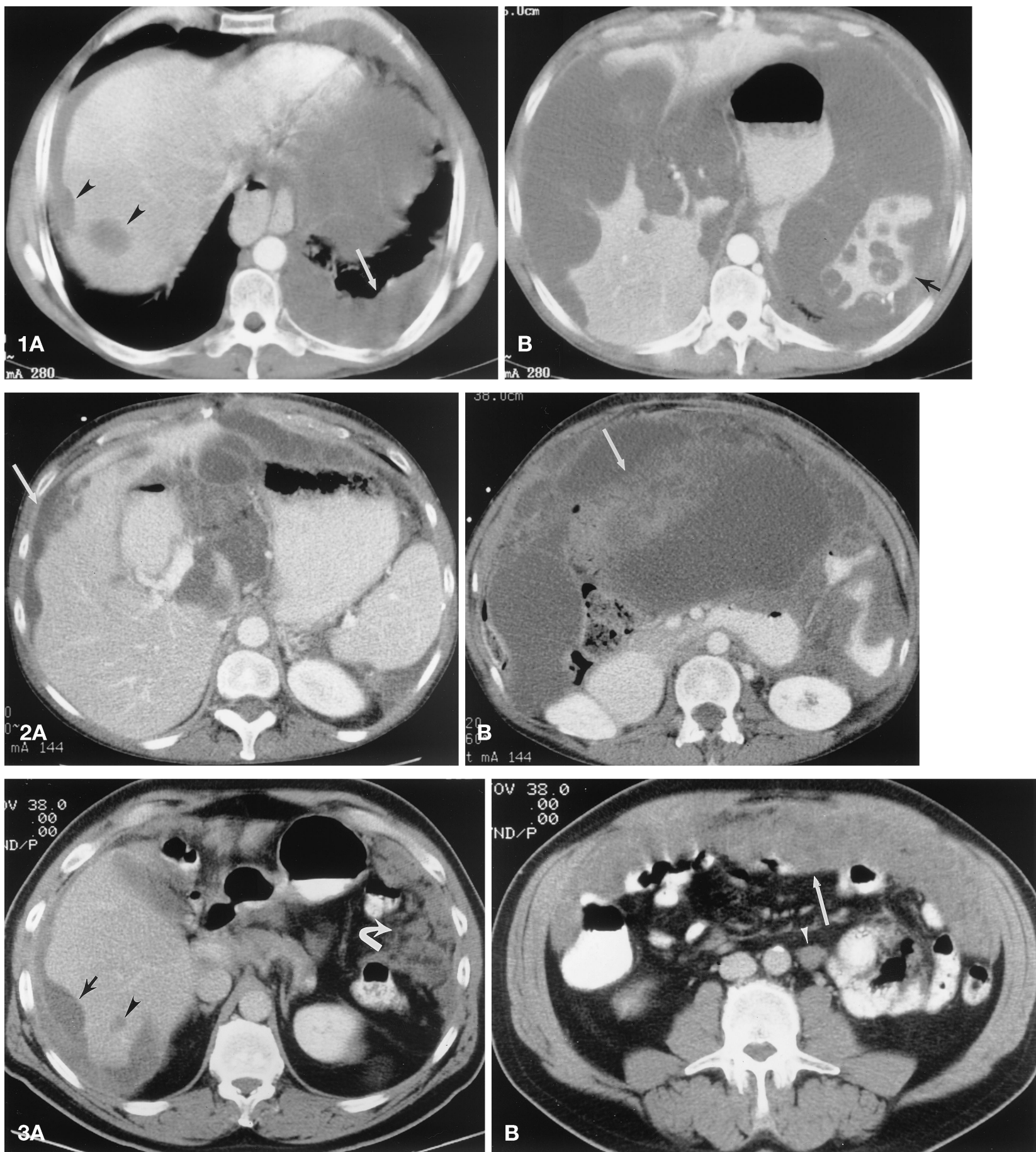

Fig. 1. A 51-year-old man with PMCA. A Chest CT shows left pleural effusion (arrow). Two low-attenuation implants (arrowheads) are found in the liver. B A lower image shows a large amount of fluid or mucin in the abdomen. Multiple low-attenuation implants (arrow) are seen in the spleen, indicating parenchymal invasion.

Fig. 2. A 64-year-old woman with DPAM. A Abdominal CT shows a large amount of fluid or mucin around the spleen and liver, with typical scalloping (arrow). B A lower image shows extensive amount of fluid and a complex cystic mass (arrow) in the midabdomen measuring $16 \times 25.5 \mathrm{~cm}$. The fluid extends around the liver and

spleen and displaces the bowel.

Fig. 3. A 55-year-old man with PMCA. A CT shows nodular carcinomatosis surrounding the liver and casting invagination (arrow) on the surface of the liver. A small $(1 \mathrm{~cm})$ hypodense lesion (arrowhead) in the liver indicates hepatic parenchymal invasion. Nodular omental infiltration is also present (curved arrow). B A lower image shows massive carcinomatosis surrounding the organs and omental caking in the abdomen (arrow). A left periaortic lymph node with a diameter of $1.5 \mathrm{~cm}$ (arrowhead) is present below the level of the duodenum. 

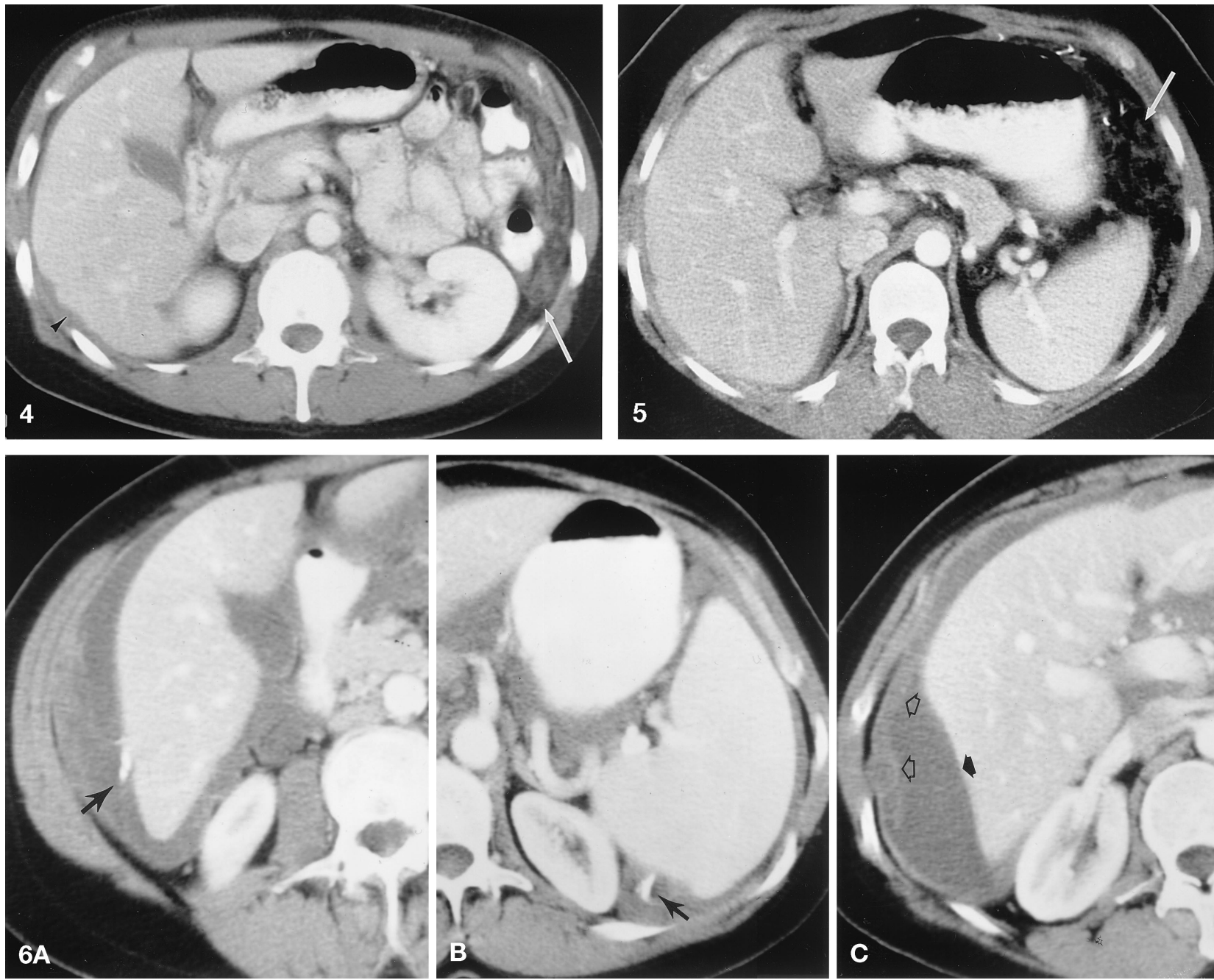

Fig. 4. A 36-year-old woman with PMCA. CT shows soft tissue infiltration (arrow) in the left upper quadrant, indicating interstitial infiltration of the peritoneum. Minimal hepatic scalloping (arrowhead) is present.

Fig. 5. A 47-year-old woman with DPAM. CT shows soft tissue infiltration (arrow) in the left upper quadrant surrounding the stomach and spleen, indicating interstitial reticular infiltration of the peritoneum.

mucin are not associated with epithelium. Epithelial cells are benign nuclei typical of adenomatous changes without definite carcinomatous findings and have little mitotic activity. Histologic criteria of PMCA include mucin in association with more abundant epithelium, nuclear hyperchomatism, and a high nuclear-to-cytoplasmic ratio. Nucleoli are enlarged and irregular. This categorization provides the means for understanding the expected behavior of diffuse peritoneal tumor and of pseudomyxoma peritonei in particular. Whereas most radiologists are familiar with peritoneal carcinomatosis, DPAM has not received similar attention.

Without an understanding of the existence of DPAM and its features, it may be easy to assume that all perito-
Fig. 6. A 45-year-old woman with DPAM. A, B CTs show a small calcified lesion (arrow in A) associated with soft tissue adjacent to the liver and a small calcified peritoneal mass (arrow in B) adjacent to the posterior aspect of the spleen. C CT shows a fluid collection (solid arrow) bordered by nodular implants (open arrows) along the lateral aspect of the liver.

neal diseases are more common and more ominous peritoneal carcinomatosis. Because DPAM is a histologically benign form of multifocal peritoneal mucinous tumor, its prognosis is better than that of PMCA [3]. We have noticed that patients with DPAM have many CT findings similar to those of patients with PMCA. Indeed, the overlap in $\mathrm{CT}$ findings may not permit an accurate prediction of the etiology in all cases. However, despite the similar manifestations of the two disease processes, there are isolated features that are sufficiently different to suggest one disease or the other.

One distinguishing finding was coexistent chest disease in one case of PMCA. This would follow from the 
fact that PMCA is a more aggressive process, exhibits malignant behavior, and is capable of metastasizing distantly outside the abdomen. One would assume that direct extension of mucinous ascites through the hemidiaphragm could also occur through pleuroperitoneal canals and that histologically benign tumor in DPAM could form in the chest, but the incidence is much less than in PMCA. This conclusion, however, is limited by our small patient population with DPAM.

Several abdominal CT findings are more common in PMCA than in DPAM and vice versa. Although ascites occurs in both, in this series, a larger volume of mucinous ascites occurred in DPAM. This observation correlates with the assumption that pseudomyxoma peritonei occurs in DPAM and presents as massive volumes of gelatinous peritoneal deposits. Solid omental mass lesions occur only in PMCA. This probably also relates to the more aggressive behavior of PMCA, in which the distribution of solid mass lesions of the peritoneum occurs diffusely. Lymphadenopathy and the presence of a primary malignant lesion are commonly seen in patients with PMCA. Calcification in masses occurs somewhat more commonly in DPAM than in PMCA. The etiology of this calcification in DPAM has not been elucidated. Further, calcification in psammoma bodies in carcinomatosis is known to occur. Therefore, this cannot be considered a clear discriminating feature between the two disease processes.

CT findings in the abdomen overlapped considerably, reflecting the fact that the peritoneum is primarily affected in both diseases. Hepatic scalloping and parenchymal invasions were commonly seen in both DPAM and PMCA [8, 9]. Peritoneal implants and mesenteric interstitial infiltration were seen in both DPAM and PMCA despite the different clinical course [8-10].

In conclusion, DPAM is an uncommonly considered radiologic diagnosis. DPAM is characterized by peritoneal lesions with benign epithelium, with or without an associated appendiceal mucinous adenoma. Pseudomyxoma peritonei is characterized by mucinous ascites, noninvasive mucinous implants, and histologically benign epithelium. DPAM is a clinicopathologic entity distinct from PMCA, with different histologic and gross features and prognosis.
Although there is great overlap in CT features between the two diseases, there are some features that separate the two. This difference especially includes in DPAM the lack of omental cake. Our small series suggests that in DPAM there is a lack of lymphadenopathy and a higher prevalence of calcification. However, because DPAM is relatively rare, we do not have enough data to establish that these differences are statistically significant, and a larger series would be required to establish whether or not there are any significant differences. It may be valuable for radiologists to be familiar with the existence of DPAM to properly evaluate the patient with pseudomyxoma peritonei, otherwise presumed to arise from malignant multifocal peritoneal tumor such as PMCA, who survive over a relatively long time.

\section{References}

1. Ronnett BM, Zahn CM, Kurman RJ, et al. Disseminated peritoneal adenomucinosis and peritoneal mucinous carcinomatosis. Am J Surg Pathol 1995;19:1390-1408

2. Ronnett BM, Shmookler BM, Sugarbaker PH, et al. Pseudomyxoma peritonei: new concept in diagnosis, origin, nomenclature, and relationship to mucinous borderline (low malignant potential) tumor of the ovary. Anat Pathol 1997;2:197-226

3. Wirtzfeld DA, Rodriguez-Bigas M, Weber T, et al. Disseminated peritoneal adenomucinosis: a critical review. Ann Surg Oncol 1999; 6:797-801

4. Higa E, Rosai J, Pizzimbono CA, et al. Mucosal hyperplasia, mucinous cystadenoma, and mucinous cystadenocarcinoma of the appendix: a re-evaluation of appendiceal "mecocele." Cancer 1973; 32:1525-1541

5. Krebs TL, Daly BD, Wong-You-Cheong J, et al. General case of the day. RadioGraphics 1998;18:1049-1050

6. Cheng KK. Experimental study of mucocele of appendix and pseudomyxoma peritonei. J Pathol Bacteriol 1949;116:439-443

7. Woodruff R, McDonald JR. Benign and malignant cystic tumors of the appendix. Surg Gynecol Obstet 1940;71:750-755

8. Papadatos D, Taourel P, Bret PM. CT of leiomyomatosis peritonealis disseminata mimicking peritoneal carcinomatosis. AJR 1996; 167:475-476

9. Abulafia O, Angel C, Sherer DM, et al. Computed tomography of leiomyomatosis peritonealis disseminata with malignant transformation. Am J Obstet Gynecol 1993;169:52-54

10. Tsai CJ, Lee HH. Disseminated peritoneal adenomucinosis-a diagnostic approach by peritoneoscopy. Gastrointest Endosc 1998;48: 312-314 